\title{
Hearing Scenes: A Neuromagnetic Signature of Auditory Source and Reverberant Space Separation
}

\author{
(D) Santani Teng, ${ }^{1}$ Verena R. Sommer, ${ }^{1,2}$ DDimitrios Pantazis, ${ }^{3}$ and Aude Oliva ${ }^{1}$
}

DOI:http://dx.doi.org/10.1523/ENEURO.0007-17.2017

${ }^{1}$ Computer Science and Artificial Intelligence Laboratory, Massachusetts Institute of Technology, Cambridge, MA 02139, ${ }^{2}$ Amsterdam Brain and Cognition Centre, University of Amsterdam, 1018 WS Amsterdam, The Netherlands, and ${ }^{3}$ McGovern Institute for Brain Research, Massachusetts Institute of Technology, Cambridge, MA 02139

\begin{abstract}
Perceiving the geometry of surrounding space is a multisensory process, crucial to contextualizing object perception and guiding navigation behavior. Humans can make judgments about surrounding spaces from reverberation cues, caused by sounds reflecting off multiple interior surfaces. However, it remains unclear how the brain represents reverberant spaces separately from sound sources. Here, we report separable neural signatures of auditory space and source perception during magnetoencephalography (MEG) recording as subjects listened to brief sounds convolved with monaural room impulse responses (RIRs). The decoding signature of sound sources began at $57 \mathrm{~ms}$ after stimulus onset and peaked at $130 \mathrm{~ms}$, while space decoding started at $138 \mathrm{~ms}$ and peaked at $386 \mathrm{~ms}$. Importantly, these neuromagnetic responses were readily dissociable in form and time: while sound source decoding exhibited an early and transient response, the neural signature of space was sustained and independent of the original source that produced it. The reverberant space response was robust to variations in sound source, and vice versa, indicating a generalized response not tied to specific source-space combinations. These results provide the first neuromagnetic evidence for robust, dissociable auditory source and reverberant space representations in the human brain and reveal the temporal dynamics of how auditory scene analysis extracts percepts from complex naturalistic auditory signals.
\end{abstract}

Key words: audition; auditory scene analysis; magnetoencephalography; multivariate pattern analysis; reverberation

\section{Significance Statement}

We often unconsciously process echoes to help navigate places or localize objects. However, very little is known about how the human brain performs auditory space analysis and, in particular, segregates direct sound-source information from the mixture of reverberant echoes that characterize the surrounding environment. Here, we used magnetoencephalography (MEG) to characterize the time courses of auditory source and space perception in the human brain. We found that the brain responses to spatial extent in reverberant environments were separable from those to the sounds that produced the reverberations and robust to variations in those sounds. Our results demonstrate the existence of dedicated neural mechanisms that separately process auditory reverberations and sources within the first few hundred milliseconds of hearing. 


\section{Introduction}

Imagine walking into a cathedral at night. Even in darkness, the passage from the narrow entryway to the large nave is immediately apparent. The reverberations produced by multiple echoes of footfalls, speech, and other sounds produce a percept of the surrounding space, distinct from the sound sources that created the echoes.

Much prior work in audition has investigated the spatial localization and perceptual organization of sound sources (Middlebrooks and Green, 1991; Bregman, 1994; Blauert, 1997; Bizley and Cohen, 2013). The surrounding environment of a sound source (i.e., the various surfaces from which reverberant reflections arise) has primarily been characterized by its effects on sound-source perception. The auditory system typically works to counteract the distorting effects of reverberation from interior surfaces, facilitating perceptual robustness of stimulus spatial position (Litovsky et al., 1999; Shinn-Cunningham, 2003; Devore et al., 2009; Brown et al., 2015), speaker identity (Brandewie and Zahorik, 2010; Watkins and Raimond, 2013), or estimated loudness (Stecker and Hafter, 2000; Zahorik and Wightman, 2001). Neurons in the auditory cortex have been shown to represent denoised or dereverberated versions of speech sounds even when presented under those distorting conditions (Mesgarani et al., 2014). Yet beyond being an acoustic nuisance to overcome, reverberations themselves provide informative cues about the environment. Humans perceive cues such as the ratio of direct to reverberant acoustic energy (DRR) to estimate sound-source distances (Mershon and King, 1975; Bronkhorst and Houtgast, 1999; Zahorik, 2001; Zahorik et al., 2005; Kolarik et al., 2016), and reverberation time (RT, a measure of reverberant energy decay) to estimate the sizes of enclosed rooms (McGrath et al., 1999; Hameed et al., 2004; Pop and Cabrera, 2005; Ca-

Received December 31, 2016; accepted February 6, 2017; First published February 13, 2017.

The authors declare no competing financial interests.

Author contributions: S.T., D.P., and A.O. designed research; S.T., V.R.S., and D.P. performed research; S.T. and D.P. analyzed data; S.T., D.P., V.R.S., and A.O. wrote the paper.

This work was supported by National Eye Institute Grant EY020484 (to A.O.), the Vannevar Bush Faculty Fellowship program sponsored by the Basic Research Office of the Assistant Secretary of Defense for Research and Engineering and funded by the Office of Naval Research Grant N00014-16-1-3116 (to A.O.), and the McGovern Institute Neurotechnology Program (to A.O. and D.P.).

Acknowledgments: We thank James Traer, Joshua McDermott, and Radoslaw Cichy for assistance in stimulus design and helpful comments on earlier versions of this manuscript. MEG scanning was conducted at the Athinoula A. Martinos Imaging Center at the McGovern Institute for Brain Research, Massachusetts Institute of Technology. Stimuli and data used in this study are available on request. V.R.S. is currently affiliated with the Max Planck Institute for Human Development (Berlin, Germany) and the International Max Planck Research School on the Life Course.

Correspondence should be addressed to Santani Teng, Massachusetts Institute of Technology, 77 Massachusetts Avenue, 32-D430, Cambridge, MA 02139, E-mail: santani@mit.edu.

DOI:http://dx.doi.org/10.1523/ENEURO.0007-17.2017

Copyright (C) 2017 Teng et al.

This is an open-access article distributed under the terms of the Creative Commons Attribution 4.0 International, which permits unrestricted use, distribution and reproduction in any medium provided that the original work is properly attributed. brera and Pop, 2006; Lokki et al., 2011; Kaplanis et al., 2014).

Direct and reflected environmental sounds usually arrive at the ear in a single stream; their perceptual separation is an underdetermined computational problem whose resolution by the auditory system remains unclear. Recent behavioral work suggests that the auditory system performs a scene analysis operation in which natural reverberation is separated from the originating sound source and analyzed to extract environmental information (Traer and McDermott, 2016). The neural basis of that operation, however, remains largely unexplored.

Here, to investigate the auditory coding of environmental space in the human brain, we recorded magnetoencephalography (MEG) responses to auditory stimuli comprising sounds enclosed by reverberant spaces. We operationalized spaces as the auditory room impulse response (RIR) of real-world spaces of different spatial extent (small to large rooms). The stimuli were constructed by convolving brief anechoic impact sounds of different objects with the spatial RIRs, allowing us to vary spatial extent and type of source independently. We hypothesized that both the sound source and the type of spaces could be separably decoded from the neural responses to naturalistic reverberant sounds. We found that neuromagnetic responses to spatialized sounds were readily dissociable into representations of the source and its reverberant enclosing space and that these representations were robust to environmental variations. Our MEG results constitute the first neuromagnetic marker of auditory spatial extent, dissociable from sound-source discrimination, suggesting that sound sources and auditory space are processed discretely in the human brain.

\section{Materials and Methods}

We conducted two MEG experiments. Experiment 1 aimed to investigate whether auditory space and source representations are encoded in MEG signals and whether they are dissociable from each other. Experiment 2 was a control study examining whether neural representations reflect the timing of neural operations or low-level stimulus properties.

\section{Experiment 1: separating MEG signatures of sound sources and reverberant spaces \\ Participants}

We recruited 14 healthy volunteers (nine females, age mean $\pm S D=27.9 \pm 5.2$ years) with self-reported normal hearing and no history of neurologic or psychiatric disease. Participants were compensated for their time and provided informed consent in accordance with guidelines of the MIT Committee on the Use of Humans as Experimental Subjects (COUHES).

\section{Stimuli}

Stimuli were recordings of three different brief monaural anechoic impact sounds (hand pat, pole tap, and ball bounce), averaging $176 \mathrm{~ms}$ in duration. Each sound was convolved with three different monaural RIRs corresponding to real-world spaces of three different sizes, yielding a total of nine spatialized sound conditions. The RIRs were selected from a set described in detail in (Traer and 
McDermott, 2016). Briefly, the RIRs were measured by recording repeated Golay sequences broadcast from a portable speaker and computing the impulse response from the averaged result (Zhou et al., 1992). The use of Golay sequences allowed ambient and transient noise from busy real-world sites to be averaged out of the final response. The speaker-recorder relationship was constant at $\sim 1.5 \mathrm{~m}$ straight ahead; thus, differences between IRs did not encode variations in egocentric spatial position (azimuth, elevation, or distance) relative to the virtual sound sources in our stimuli. Rooms consisted of three real-world everyday spaces, a kitchen, a hallway, and a gym, with estimated volumes (based on room boundary dimensions) of $\sim 50,130$, and $600 \mathrm{~m}^{3}$. Reverberation times $\left(\mathrm{RT}_{60}\right.$, the time for an acoustic signal to drop by 60 $\mathrm{dB}$ ) of the small-, medium-, and large-space RIRs were $0.25,0.51$, and $0.68 \mathrm{~s}$, respectively, averaged across frequencies from $20 \mathrm{~Hz}$ to $16 \mathrm{kHz}$.

\section{MEG testing protocol}

We presented stimuli to participants diotically through tubal-insert earphones (Etymotic Research, Elk Grove Village) at a comfortable volume, $\sim 70 \mathrm{~dB}$ SPL. Stimulus conditions were presented in random order (Psychophysics Toolbox; RRID: SCR_002881) with stimulus onset asynchronies (SOAs) jittered between 2000 and $2200 \mathrm{~ms}$. Every three to five trials (four on average), a deviant vigilance target (brief speech sound) was presented, prompting participants to press a button and blink. SOAs between vigilance target and the following stimulus were $2500 \mathrm{~ms}$. Target trials were excluded from analysis. Each experimental session lasted $\sim 65 \mathrm{~min}$ and was divided into 15 runs containing 10 trials from each condition, for a total of 150 trials per condition in the entire session.

\section{Behavioral testing protocol}

In our MEG scanning protocol, we used a passivelistening paradigm to avoid contamination of brain signals with motor-response artifacts. Thus, to test explicit perceptual judgments of the auditory scene stimuli, we conducted separate behavioral tests of space and soundsource discrimination. Participants $(N=14)$ listened to sequential pairs of the stimuli described above, separated by $1500-m s$ SOA. In separate blocks, participants made speeded same-different judgments on the sound sources or spaces in the stimulus pairs. Condition pairs and sequences were counterbalanced for each participant, and the order of source- and space-discrimination blocks was counterbalanced across participants. Over the course of four blocks lasting $\sim 40 \mathrm{~min}$, participants completed a total of 36 trials per category. We collected reaction time and accuracy data from participants' responses.

\section{MEG data acquisition}

MEG recordings were obtained with an Elekta Neuromag TRIUX system (Elekta), with continuous whole-brain data acquisition at $1 \mathrm{kHz}$ from 306 sensors (204 planar gradiometers; 102 magnetometers), filtered between 0.3 and $330 \mathrm{~Hz}$. Head motion was continuously tracked through a set of five head-position indicator coils affixed to the participant's head.

\section{MEG preprocessing and analysis}

Data were motion compensated and spatiotemporally filtered offline (Taulu et al., 2004; Taulu and Simola, 2006) using Maxfilter software (Elekta). All further analysis was conducted using a combination of Brainstorm software (Tadel et al., 2011; RRID: SCR_001761) and Matlab (Natick; RRID: SCR_001622) in-house analysis scripts. We extracted epochs for each stimulus presentation with a 200 -ms prestimulus baseline and 1000 -ms poststimulus onset, removed the baseline mean from each sensor, and applied a $30-\mathrm{Hz}$ low-pass filter.

\section{MEG multivariate analysis}

To determine the time course of reverberant space and source discrimination, we analyzed MEG data using a linear support vector machine (SVM) classifier (Chang and Lin, 2011; RRID:SCR_010243; http://www.csie.ntu.edu.tw/ cjlin/libsvm/). For each time point $t$, the MEG sensor data were arranged in a 306-dimensional pattern vector for each of the $M=150$ trials per condition (Fig. $1 B$ ). To increase SNR and reduce computational load, the $M$ single-trial pattern vectors per condition were randomly subaveraged in groups of $k=10$ to yield $M / k$ subaveraged pattern vectors per condition. We then used a leaveone-out cross-validation approach to compute the SVM classifier performance in discriminating between every pair of conditions. The whole process was repeated $\mathrm{K}=$ 100 times, yielding an overall classifier decoding accuracy between every pair of conditions for every time point $t$ (Fig. 1B).

The decoding accuracies were then arranged into $9 \times 9$ representational dissimilarity matrices (RDMs; Kriegeskorte et al., 2008), one per time point $t$, indexed by condition and with the diagonal undefined. To generate the single-sound decoding time course (Fig. 1C), a mean accuracy was computed from the individual pairwise accuracies of the RDM for each time point.

For reverberant space decoding, conditions were pooled across the three sound-sources, resulting in $3 \mathrm{M}$ trials for each space. An SVM classifier was trained to discriminate between every pair of spaces and results were averaged across all three pairs. Decoding procedures were similar as above, but subaveraging was increased to $\mathrm{k}=30$ and repetitions to $\mathrm{K}=300$. For soundsource decoding, we pooled across the three spaces and performed the corresponding analyses (Fig. 2A).

Statistical significance of MEG decoding time courses was determined with permutation tests against the null hypothesis of chance-level (50\%) MEG decoding accuracy. For each of 1000 permutations, each time point per participant was randomly multiplied by +1 or -1 to produce an empirical distribution of decoding accuracies from which $p$ values could be derived. Cluster-size inference (Maris and Oostenveld, 2007) was used to control for multiple comparisons, with the cluster-definition threshold set at $p=0.05$ (one-sided). Clusters were reported based on exceeding the $95 \%$ of the maximal cluster-size distribution. 95\% confidence intervals (Cls) for onset and peak latencies were determined by bootstrapping the participants 1000 times and repeating the analysis to obtain empirical distributions. 
A Sound source

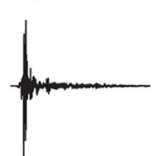

18)) Hand pat

22)) Pole tap

3)) Ball bounce
Room impulse response
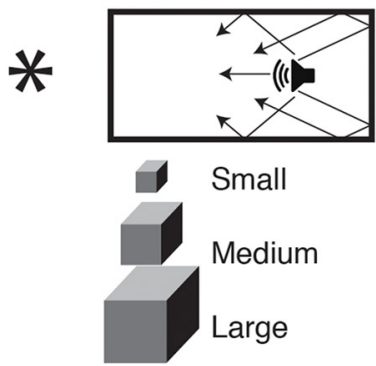

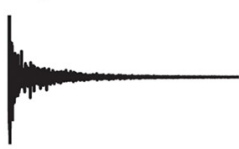

$\mathrm{RT}_{60}=.25 \mathrm{~s}, \mathrm{vol} \approx 50 \mathrm{~m}^{3}$

$\mathrm{RT}_{60}=.51 \mathrm{~s}, \mathrm{vol} \approx 130 \mathrm{~m}^{3}$

$\mathrm{RT}_{60}=.68 \mathrm{~s}, \mathrm{vol} \approx 600 \mathrm{~m}^{3}$
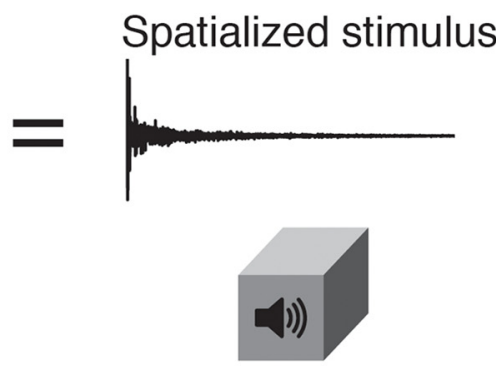

9 conditions

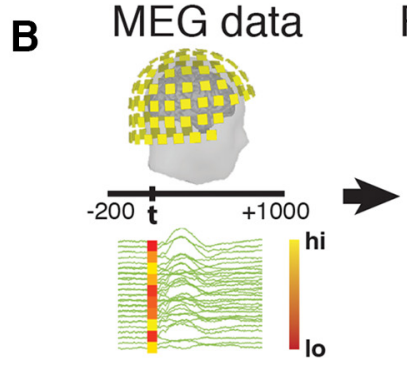

$306 \times 1$ vector per time point $t$
Pairwise classification

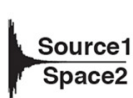

VS.

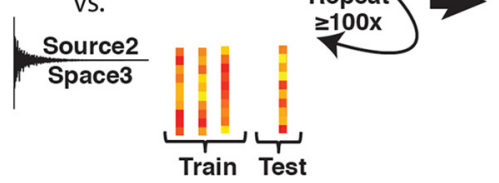

Leave-one-out cross-validation with randomly subaveraged vectors
Decoding matrix

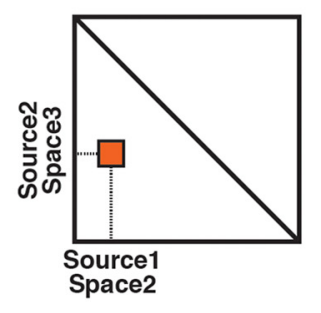

Representational dissimilarity matrix (RDM) All pairwise decoding accuracies for time $t$

\section{Classification time course}

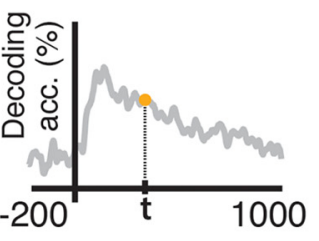

Average across RDM for time $t$

\section{Decoding individual conditions}
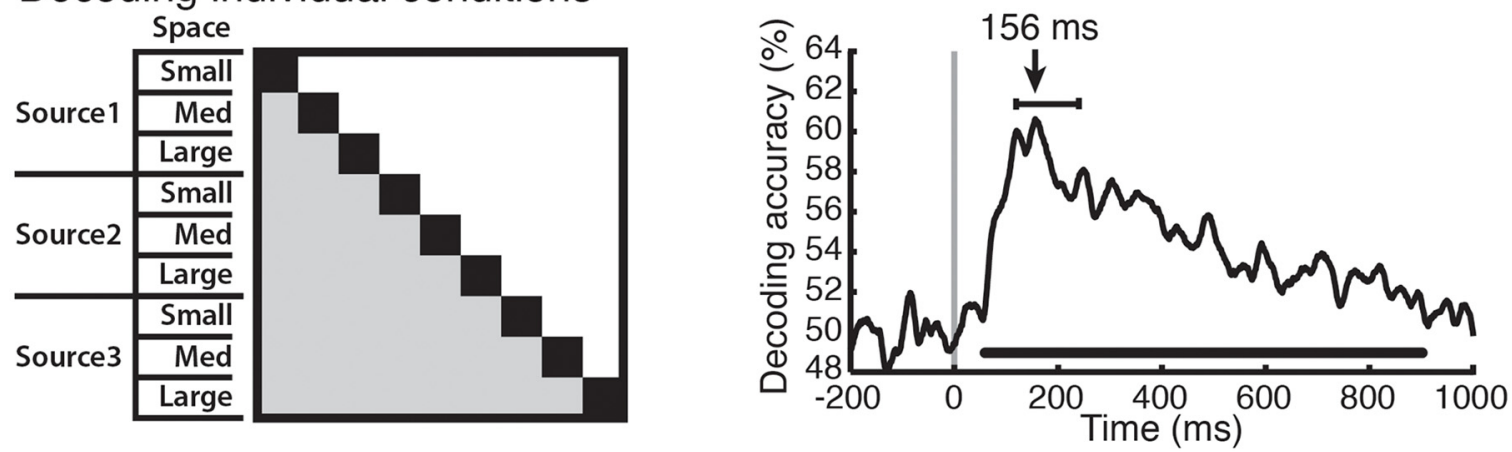

Figure 1. Stimulus conditions, MEG classification scheme, and single-sound decoding time course. A, Stimulus design. Three brief sounds were convolved with three different RIRs to produce nine sound sources spatialized in reverberant environments. $\boldsymbol{B}$, MEG pattern vectors were used to train an SVM classifier to discriminate every pair of stimulus conditions (three sound sources in three different space sizes each). Decoding accuracies across every pair of conditions were arranged in $9 \times 9$ decoding matrices, one per time point $t$. $\boldsymbol{C}$, Averaging across all condition pairs (shaded matrix partition) for each time point $t$ resulted in a single-sound decoding time course. Lines below time course indicates significant time points $(N=14$, cluster-definition threshold, $p<0.05$, 1000 permutations). Decoding peaked at $156 \mathrm{~ms}$; error bars represent $95 \% \mathrm{Cl}$.

\section{MEG cross-classification analysis}

To determine the robustness of space size and soundsource representations to environmental variation, we performed a cross-classification analysis in which different orthogonal experimental factors were assigned to training and testing sets. For example, the cross-classification of reverberant space (Fig. 2B) was conducted by training the SVM classifier to discriminate spaces on two sound sources and testing it on the third sound source. This analysis was repeated for all such train-test combinations and the results were averaged to produce the final cross-classification accuracy plots. SVM decoding was performed similarly to the single-condition analyses, but the training set had $2 \mathrm{M}$ trials, subaveraging was set to $\mathrm{k}=$
20 , and repetitions to $K=150$. Cross-classification of sound-source identity across space sizes was performed with corresponding analyses.

\section{MEG spatial (sensorwise) analysis}

The above analyses used signals from the entire suite of MEG sensors to maximize information for decoding sources and spaces. To characterize the spatial distribution of the decoding time course, we conducted a sensorwise analysis of the MEG-response patterns. Specifically, since the 306 MEG sensors are physically arranged in 102 triplets (each triplet consisting of one magnetometer and two gradiometers in the same location), we repeated the multivariate analyses described 
A Reverberant space

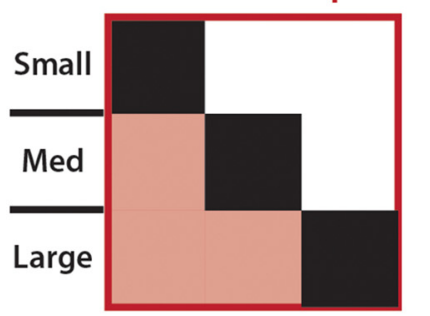

\section{Sound source}

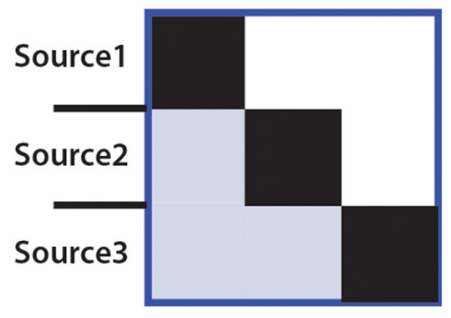

B
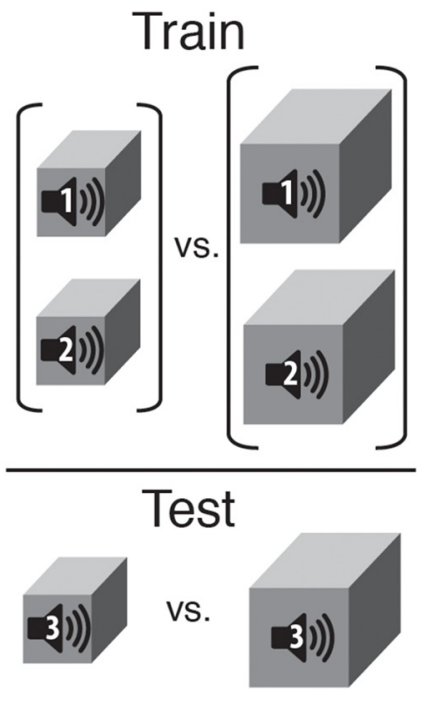
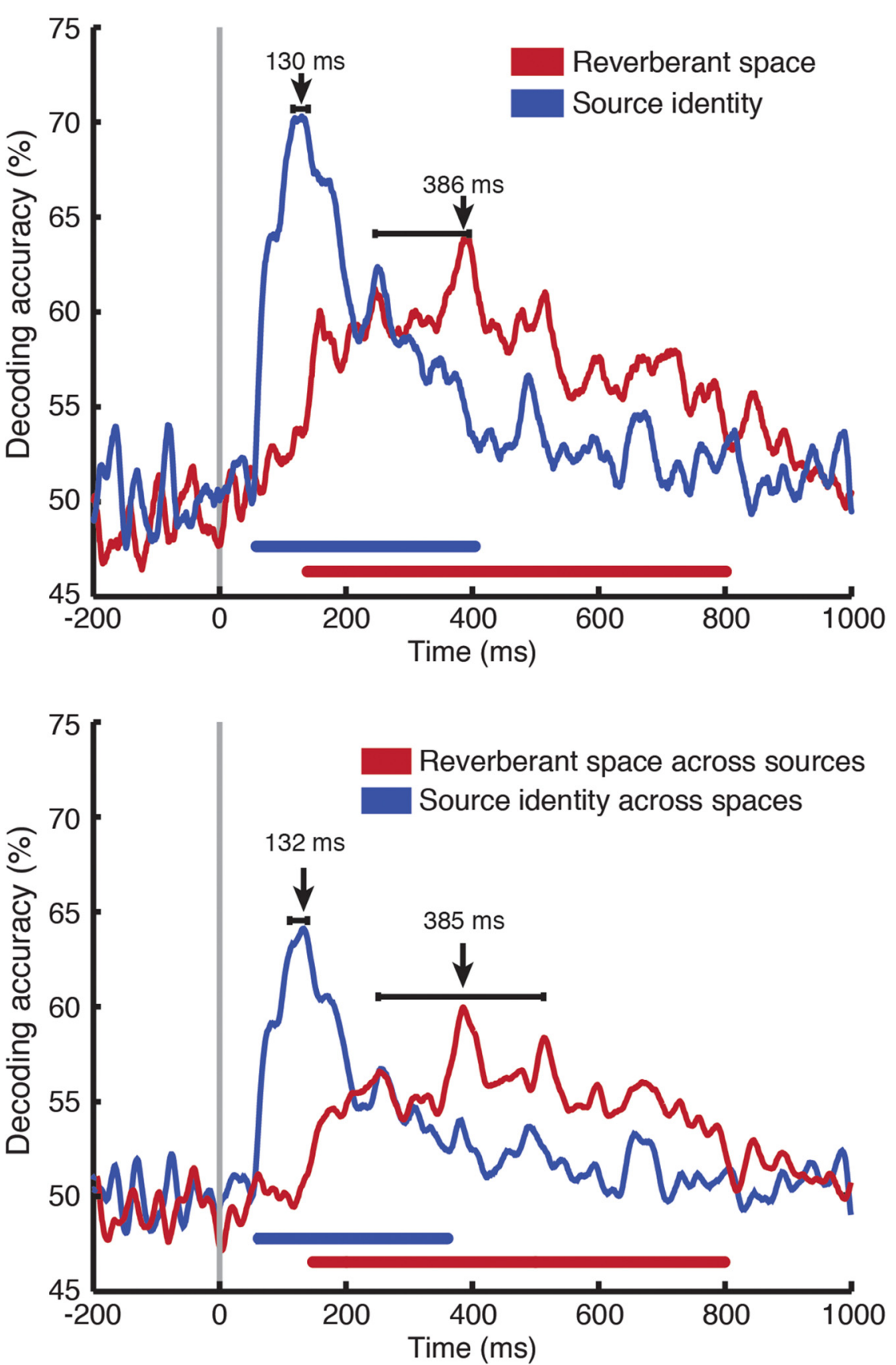

Figure 2. Separable space and source identity decoding. $\boldsymbol{A}$, Individual conditions were pooled across source identity (left, top) or space size (left, bottom) in separate analyses. Classification analysis was then performed on the orthogonal stimulus dimension to establish the time course with which the brain discriminated between space (red) and source identity (blue). Sound-source classification peaked at $130 \mathrm{~ms}$, while space classification peaked at $386 \mathrm{~ms}$. Significance indicators and latency error bars on plots same as in Figure 1. B, Space was classified across sound sources and vice versa. Left panel, Cross-classification example in which a classifier was trained to discriminate between spaces on sound sources 1 and 2, then tested on space discrimination on source 3. Right panel, Sound-source cross-classification example in which a classifier was trained to discriminate between sound sources on space sizes 1 and 2 , then tested on sound-source discrimination on space 3. $\boldsymbol{B}$, Results from all nine such pairwise train-test combinations were averaged to produce a classification time course in which the train and test conditions contained different experimental factors. Sound-source cross-classification peaked at $132 \mathrm{~ms}$, while space cross-classification peaked at 385 ms. Significance bars below time courses and latency error bars same as in Figure 1.

above at each of the 102 sensor locations but using a three-dimensional (rather than 306-dimensional) pattern vector for each location. This yielded a $9 \times 9$ RDM of pairwise classification accuracies at each sensor location and at each time point. Thus, rather than the single wholebrain decoding time course shown in Figures 1 and 2, we generated 102 decoding time courses, one for each sensor triplet location, visualized as sensor maps (Fig. 3). 
A Sound source decoding, $130 \mathrm{~ms}$

B Reverberant space decoding, $385 \mathrm{~ms}$
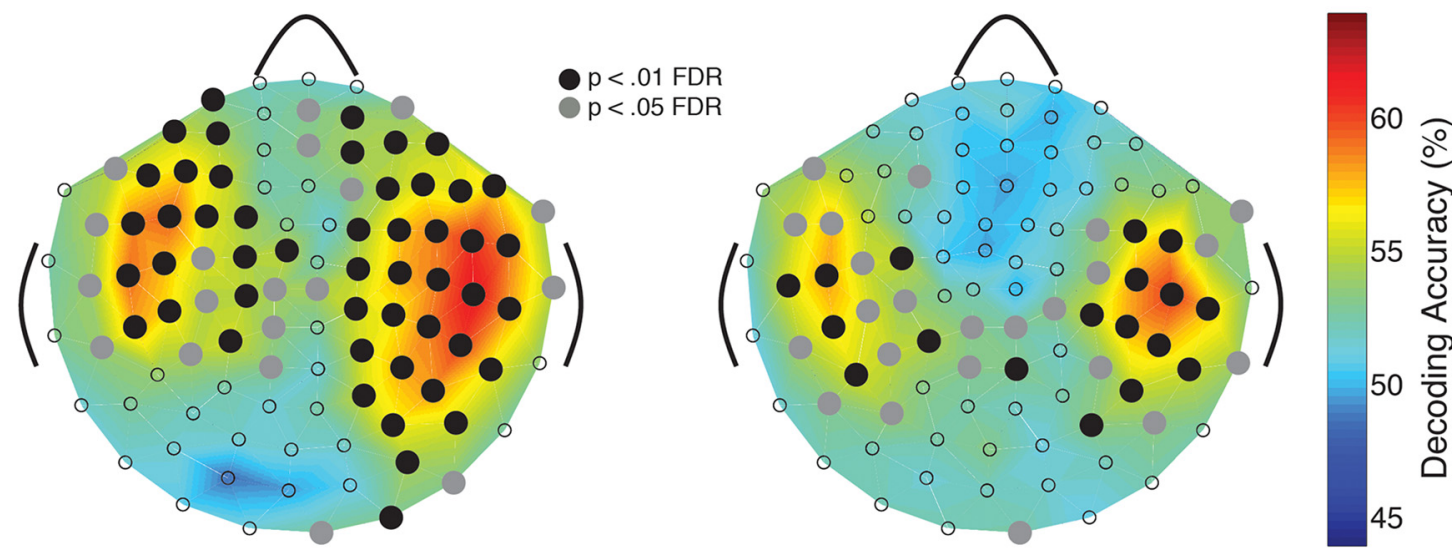

Figure 3. Sensorwise decoding of source identity and space size. MEG decoding time courses were computed separately for 102 sensor locations yielding decoding sensor maps. $\boldsymbol{A}$, Sensor map of sound source decoding at the peak of the effect (130 ms). $\boldsymbol{B}$, Sensor map of space size decoding at the peak of the effect $(386 \mathrm{~ms})$. Significant decoding is indicated with a black circle over the sensor position $(p<0.01$; corrected for false discovery rate (FDR) across sensors and time).

Statistically significant decoding accuracies were determined via permutation analysis $(N=14$, sign permutation test with 1000 samples, $p<0.01$, corrected for FDR across sensor positions at each time point).

\section{MEG temporal generalization analysis}

The above analyses trained and tested each SVM classifier at a unique time point, which necessarily limits information about the persistence or transience of neural representations. Thus, to further interrogate the temporal dynamics of reverberant space and source identity processing, we generalized the above analysis by training each classifier at a given time point $t$ and testing against all other time points $t$ '. This yielded a two-dimensional temporal generalization ("time-time") matrix, where the $x$ and $y$-axes index the training and testing time points, respectively, of the classifiers (Fig. 4; King and Dehaene, 2014). Statistical significance maps for each matrix were generated via $t$ test across participants for each time-time coordinate, $p<0.05$, FDR corrected.

\section{Analysis of stimulus properties}

We generated time-frequency cochleograms from each stimulus using a Matlab-based toolbox (Slaney, 1998; Ellis, 2009) that emulates the filtering properties of the human cochlea. Each wave form was standardized to 44,100 samples and passed through a gammatone filterbank (64 subbands, center frequencies $20-20,000 \mathrm{~Hz}$ ), summing the energy within overlapping 20 -ms windows in 5 -ms steps. The cochleograms were then correlated pairwise at each time point, with 64-element pattern vectors comprising the energy per frequency subband in each 5 -ms bin. The resulting Pearson correlation coefficients were then subtracted from 1 and averaged to compute the stimulus dissimilarity measure for that time point (Fig. $6 A)$. Repeating this analysis across time points yielded the overall cochleogram-based dissimilarity curve (Fig. 6B). The same analysis was performed on conditions pooled by source identity and space size (Fig. 6C) to produce separate source and space dissimilarity time courses.
Significance of the peak mismatches with the MEG decoding peaks was determined via examination of confidence intervals from bootstrapping MEG peak latencies 10,000 times.

\section{Experiment 2: controlling for stimulus duration}

For experiment 2, we recorded MEG data while participants $(N=16)$ listened to stimuli comprising the same impact sounds as in the main experiment, but repeated 10 times at 200-ms intervals, and then convolved with the same RIRs used in the main experiment. The 2-s wave form was then linearly ramped up for $1 \mathrm{~s}$ and down for 1 $s$ to avoid strong attacks at the onset of the stimulus (Fig. $7 A$ ). Consequently, each stimulus contained its source and spatial information distributed throughout the 2000-ms repetition window. We reasoned that if peak decoding latencies reflected the neural process underlying space perception, they would not be strongly yoked to stimulus temporal structure and would thus not be strongly shifted compared with experiment 1. By contrast, MEG decoding signatures yoked to the stimulus temporal structure should not only last throughout the duration of the stimulus, but peak at $\sim 1000 \mathrm{~ms}$, the peak of the stimulus amplitude envelope.

Because of the longer stimulus duration, experimental sessions were $\sim 10$ min longer than those in the main experiment. The MEG time series extracted from the neural data spanned 2701 rather than 1201 time points, from -200 to +2500 ms relative to stimulus onset. All other parameters (organization of stimulus conditions, task, presentation procedure, significance calculations) remained the same as in experiment 1 . In computing bootstrapped peak latencies, we included only the time during which the stimulus was actively playing, between 0 and $2000 \mathrm{~ms}$.

All statistical analyses are summarized in Table 1, with superscript letters in specific Results indicating rows in the table. 
A

Temporal generalization

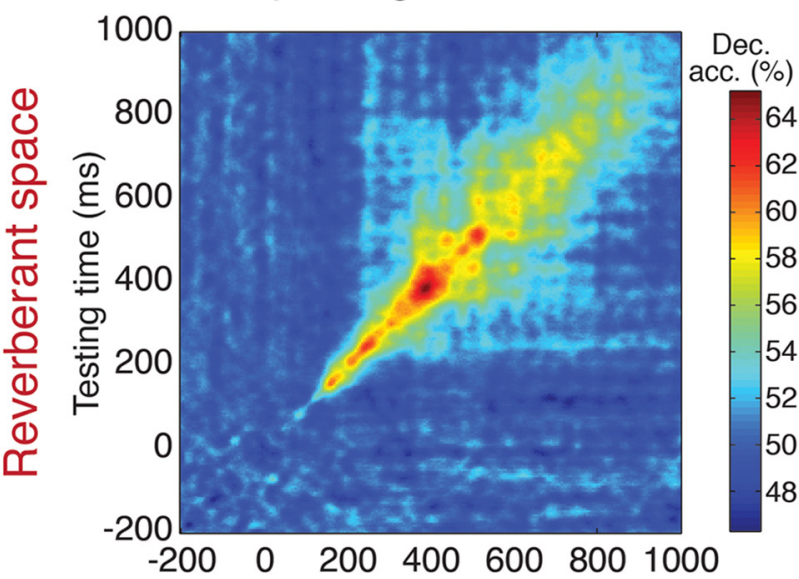

Significance
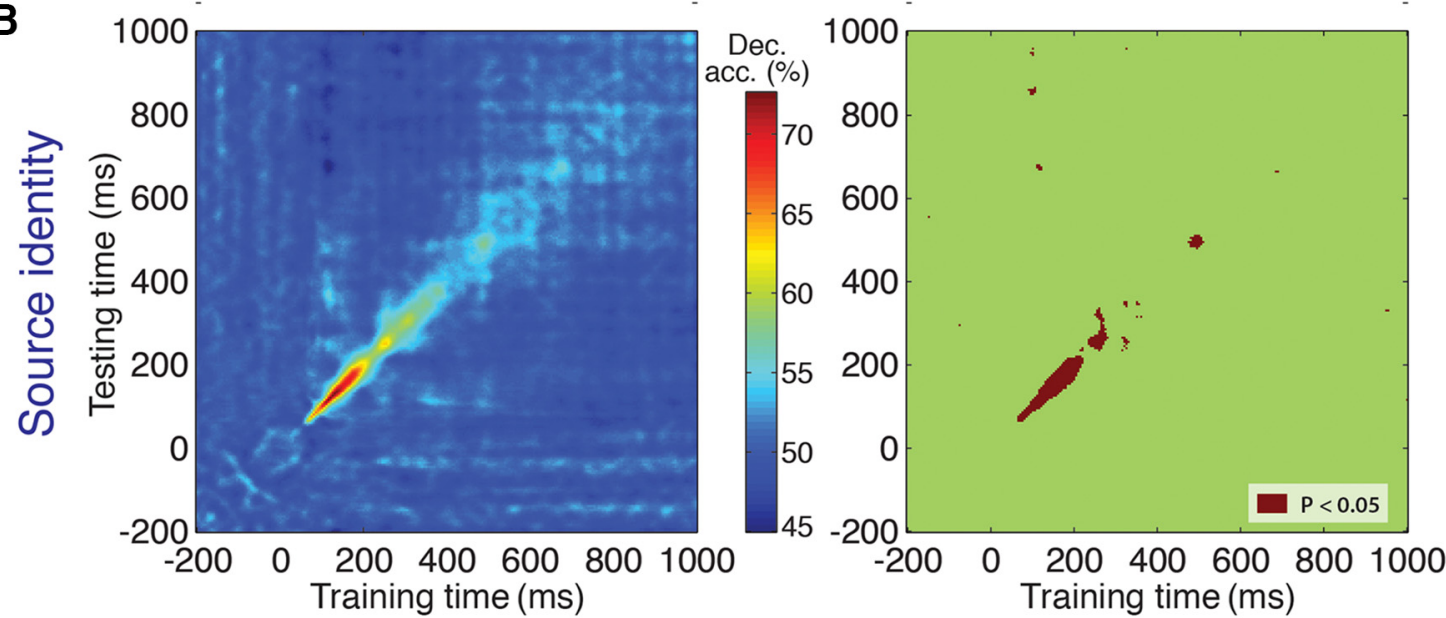

Figure 4. Temporal generalization matrix of auditory source and space decoding time courses. Left column shows the generalized decoding profiles of space $(\boldsymbol{A})$ and source $(\boldsymbol{B})$ decoding. Right column shows the statistically significant results ( $t$ test against $50 \%$, $p<0.05$, FDR corrected).

\section{Results}

\section{Experiment 1}

Auditory representations discriminated sound sources and reverberant spaces with temporally dissociable and generalizable decoding trajectories

We applied SVM to decode every pair of conditions (Fig. 1B; Carlson et al., 2013; Cichy et al., 2014, 2015). The pairwise decoding accuracies were averaged to create an overall single-condition classification time course. Classification performance increased sharply from chance levels shortly after stimulus onset, reaching significance at $59 \mathrm{~ms}(95 \% \mathrm{Cl}: 12-64 \mathrm{~ms})^{\mathrm{a}}$ and peaking at $156 \mathrm{~ms}$ $(119-240 \mathrm{~ms})^{\mathrm{b}}$. These results indicate the MEG signal was able to reliably distinguish between individual stimulus conditions.

To dissociate the neuronal dynamics of space size and sound-source discrimination, we repeated this analysis, but pooled trials across the corresponding conditions before decoding. This resulted in $3 \times 3$ RDM matrices (Fig. $2 A$, left), and averaging across the shaded regions produced the time courses of space size (red) and source identity (blue) decoding (Fig. $2 A$, right). The transient nature of source discrimination, reaching significance at 57 $\mathrm{ms}(37-60 \mathrm{~ms})^{\mathrm{c}}$ and peaking at $130 \mathrm{~ms}(116-140 \mathrm{~ms})^{\mathrm{d}}$, is in sharp contrast to the slower, sustained response of the reverberant space decoding time course, which exhibited a significantly later decoding accuracy significance onset [138 ms (71-150) ms) ${ }^{\mathrm{e}}$ ] and peak [386 ms (246-395 ms); onset latency difference, $p=0.01^{\text {g; }}$ peak latency difference, $\left.p<0.001^{\mathrm{h}}\right]$. This suggests that sound-source information is discriminated early by the auditory system, followed by reliable reverberant space discrimination. In experiment 2, we found that sources and spaces were still decodable when all stimuli were controlled for duration, suggesting that the timing is not solely dependent on stimulus duration (Fig. 8).

Stable source and reverberant space representations should be tolerant to other changing properties in a scene, such as low-level spectral differences between sound sources or spectral modulation differences between RIRs. Thus, we conducted a cross-classification analysis in which we assigned space size conditions from two sources to a training set, and the size conditions from the remaining source to a testing set (Fig. 2B, left). Results from all such train/test combinations were averaged to produce a measure of space size information generalized 
Table 1. Summary of key statistical tests

\begin{tabular}{|c|c|c|c|}
\hline Line & Data structure & Type of test & $95 \%$ confidence intervals \\
\hline a & $\begin{array}{l}\text { None assumed: classification } \\
\text { accuracy over time }\end{array}$ & $\begin{array}{l}\text { Bootstrap } N=14 \text { participants } \\
1000 \text { times to obtain empirical distribution } \\
\text { of significant decoding onset }\end{array}$ & Onset Cl: 12-64 ms \\
\hline b & $\begin{array}{l}\text { None assumed: classification } \\
\text { accuracy over time }\end{array}$ & $\begin{array}{l}\text { Bootstrap } N=14 \text { participants } \\
1000 \text { times to obtain empirical distribution } \\
\text { of significant decoding peak }\end{array}$ & Peak Cl: 119-240 ms \\
\hline C & $\begin{array}{l}\text { None assumed: classification } \\
\text { accuracy over time }\end{array}$ & $\begin{array}{l}\text { Bootstrap } N=14 \text { participants } \\
1000 \text { times to obtain empirical distribution } \\
\text { of significant sound-source decoding onset }\end{array}$ & Onset Cl: $37-60 \mathrm{~ms}$ \\
\hline d & $\begin{array}{l}\text { None assumed: classification } \\
\text { accuracy over time }\end{array}$ & $\begin{array}{l}\text { Bootstrap } N=14 \text { participants } \\
1000 \text { times to obtain empirical distribution } \\
\text { of significant sound-source decoding peak }\end{array}$ & Peak Cl: 116-140 ms \\
\hline e & $\begin{array}{l}\text { None assumed: classification } \\
\text { accuracy over time }\end{array}$ & $\begin{array}{l}\text { Bootstrap } N=14 \text { participants } \\
1000 \text { times to obtain empirical distribution } \\
\text { of significant space decoding onset }\end{array}$ & Onset Cl: $71-150 \mathrm{~ms}$ \\
\hline$f$ & $\begin{array}{l}\text { None assumed: classification } \\
\text { accuracy over time }\end{array}$ & $\begin{array}{l}\text { Bootstrap } N=14 \text { participants } \\
1000 \text { times to obtain empirical distribution } \\
\text { of significant space decoding peak }\end{array}$ & Peak Cl: 246-395 ms \\
\hline g & $\begin{array}{l}\text { None assumed: onsets of source and } \\
\text { space decoding }\end{array}$ & $\begin{array}{l}\text { Compare bootstrapped empirical distribution } \\
\text { of space decoding onset with } \\
\text { mean source decoding onset }\end{array}$ & Space onset $\mathrm{Cl}: 71-150 \mathrm{~ms}$ \\
\hline h & $\begin{array}{l}\text { None assumed: peaks of source } \\
\text { and space decoding }\end{array}$ & $\begin{array}{l}\text { Compare bootstrapped empirical distribution } \\
\text { of space decoding peak with } \\
\text { mean source decoding peak }\end{array}$ & Space peak Cl: 246-395 ms \\
\hline i & $\begin{array}{l}\text { None assumed: cross-classification } \\
\text { accuracy over time }\end{array}$ & $\begin{array}{l}\text { Bootstrap } N=14 \text { participants } 1000 \text { times } \\
\text { to obtain empirical distribution of significant } \\
\text { sound-source cross-decoding peaks }\end{array}$ & $\begin{array}{l}\text { Onset Cl: } 40-63 \mathrm{~ms} \\
\quad \text { Peak Cl: } 111-139 \mathrm{~ms}\end{array}$ \\
\hline j & $\begin{array}{l}\text { None assumed: cross-classification } \\
\text { accuracy over time }\end{array}$ & $\begin{array}{l}\text { Bootstrap } N=14 \text { participants } 1000 \text { times } \\
\text { to obtain empirical distribution of significant } \\
\text { space cross-decoding peaks }\end{array}$ & $\begin{array}{l}\text { Onset Cl: } 125-356 \mathrm{~ms} \\
\text { Peak Cl: } 251-513 \mathrm{~ms}\end{array}$ \\
\hline $\mathrm{k}$ & $\begin{array}{l}\text { None assumed: MEG-behavior } \\
\text { correlations }\end{array}$ & $\begin{array}{l}\text { Bootstrapping } N=14 \text { pool, } 10,000 \\
\text { iterations of Spearman correlation between } \\
\text { behavioral reaction time and MEG peak latency }\end{array}$ & Cl: .227-.895 \\
\hline I & $\begin{array}{l}\text { None assumed: MEG-behavior } \\
\text { correlations }\end{array}$ & $\begin{array}{l}\text { Bootstrapping } N=14 \text { pool, } 10,000 \text { iterations } \\
\text { of Spearman correlation between behavioral } \\
\text { accuracy and MEG peak accuracy }\end{array}$ & Cl: .325-.795 \\
\hline $\mathrm{m}$ & $\begin{array}{l}\text { None assumed: empirical distribution } \\
\text { of source decoding peak }\end{array}$ & $\begin{array}{l}\text { Compare bootstrapped empirical distribution } \\
\text { of source decoding peak with } \\
\text { source dissimilarity peak }\end{array}$ & Peak Cl: 116-140 ms \\
\hline$n$ & $\begin{array}{l}\text { None assumed: empirical distribution } \\
\text { of space decoding peak }\end{array}$ & $\begin{array}{l}\text { Compare bootstrapped empirical distribution } \\
\text { of space decoding peak with mean } \\
\text { space dissimilarity peak }\end{array}$ & Peak Cl: 246-395 ms \\
\hline o & $\begin{array}{l}\text { Normal distribution: MEG-model } \\
\text { correlations over time points }\end{array}$ & $\begin{array}{l}\text { Paired } t \text { test between } \\
\text { mean correlations }\end{array}$ & Mean difference Cl: 0.0470-0.0507 \\
\hline $\mathrm{p}$ & $\begin{array}{l}\text { None assumed: classification } \\
\text { accuracy over time }\end{array}$ & $\begin{array}{l}\text { Bootstrap } N=16 \text { participants } 1000 \text { times to } \\
\text { obtain empirical distribution of } \\
\text { significant source decoding onset }\end{array}$ & Source peak Cl: 96-312 ms \\
\hline q & $\begin{array}{l}\text { None assumed: classification } \\
\text { accuracy over time }\end{array}$ & $\begin{array}{l}\text { Bootstrap } N=16 \text { participants } 1000 \text { times } \\
\text { to obtain empirical distribution of } \\
\text { significant source decoding onset }\end{array}$ & Space peak Cl: $71-790 \mathrm{~ms}$ \\
\hline
\end{tabular}

across sound sources, with sound sources not overlapping between training and testing sets. We also performed an analogous analysis to cross-classify sound sources across spaces. The results (Fig. $2 B$, right) indicate time courses consistent with those in the pooled analysis, with source cross-decoding onset at $57 \mathrm{~ms}$ (40-63 ms), peaking at $132 \mathrm{~ms}(111-139 \mathrm{~ms})$; and space cross-decoding onset at $148 \mathrm{~ms}$ (125-136 ms), peaking at $385 \mathrm{~ms}(251-513 \mathrm{~ms})^{\mathrm{j}}$. This demonstrates that the neural representations of reverberant space and sound source are robust to variations in an orthogonal dimension.
Source identity and reverberant space were best decoded by bilateral temporal sensors

To determine the spatial distribution of the decoding response, we repeated the main analysis on sensor clusters in 102 distinct locations across the MEG helmet. This analysis revealed that the bulk of significant decoding performance $(p<0.01$, FDR corrected across sensors at each time point) was concentrated in sensors over bilateral temporal regions (Fig. 3). While the spatial interpretation of such an analysis is limited in resolution, the results implicate bilateral auditory cortical regions as gen- 

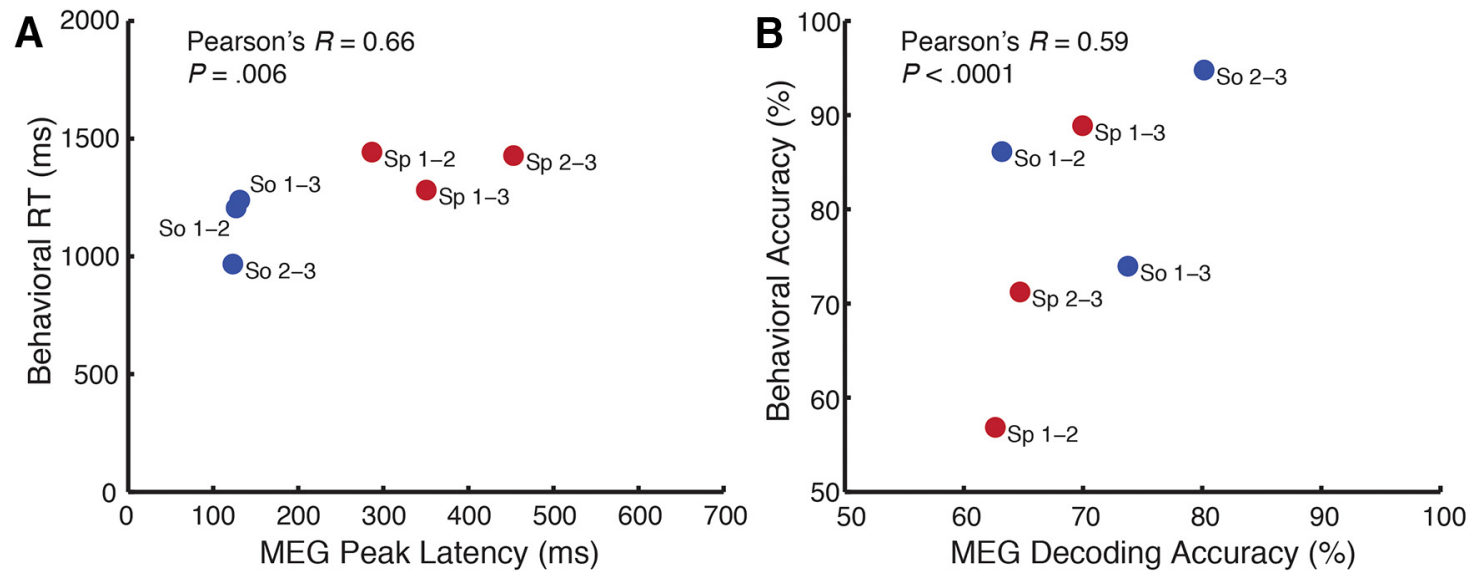

Figure 5. Behavior correlates with MEG decoding data. Assessment of linear relationships between response times and MEG peak decoding latencies $(\boldsymbol{A})$, as well as behavioral and decoding accuracies $(\boldsymbol{B})$. Bootstrapping the participant sample $(N=14, p<0.05)$ 10,000 times revealed significant correlations between RT and latency $(r=0.66, p=0.0060)$ and behavioral and decoding accuracy $(r=0.59, p<0.0001)$. Individual condition pairs are denoted by source (So; red) or space (Sp; blue) labels, with numerals indicating which conditions were compared. For space conditions: 1, small; 2, medium; 3, large. For source conditions: 1, hand pat; 2, pole tap; 3 , ball bounce.

erators of the underlying neural signals distinguishing sound source and space size information.

\section{Dynamics of reverberant space representations are slower and more sustained compared with sound- source representations}

To examine the temporal dynamics of source and space representations, we conducted a temporal generalization analysis (Cichy et al., 2014; King and Dehaene, 2014) in which a classifier trained at one time point was tested on all other time points. This produced a twodimensional matrix showing generalized decoding profiles for space and source identity (Fig. 4). The results suggest differences in processing dynamics: the narrow "diagonal chain" pattern shown for source identity decoding in Figure $4 B$ indicates that classifiers trained at a time point $t$ only generalize well to neighboring time points; by contrast, the space profile (Fig. 4A) exhibits a broader offdiagonal decoding regime, indicating that classifiers were able to discriminate between space conditions over extended time periods. This suggests that reverberant space representations are mediated by more metastable, sustained underlying neural activity, compared with transient, dynamic activity mediating sound-source representations (King and Dehaene, 2014).

MEG decoding dynamics predict relative timing and accuracy of behavioral judgments

To extract behavioral parameters that could be compared with the dynamics of the MEG signal, we binned all trials into appropriate source or space comparison categories (e.g., Space1 vs Space2, Source1 vs Source3, etc.). Within each category, we computed each participant's mean accuracy and mean response time (mean RT estimated by fitting a $\gamma$ distribution to the response time data; Palmer et al., 2011). This yielded mean accuracies and RTs in three source-comparison and three spacecomparison conditions, analogous to the pooled MEG decoding analysis. Behavioral accuracies and RTs were then correlated with MEG peak decoding accuracies and peak latencies, respectively. Significance and confidence intervals were determined by bootstrapping the behavioral and MEG participant pools 10,000 times. Behavioral RTs and peak latencies were significantly correlated $(r=$ $0.66, p=0.0060)^{\mathrm{k}}$, as were behavioral accuracies and peak decoding accuracies $(r=0.59, p<0.0001)^{\prime}$ (Fig. 5).

\section{MEG decoding peaks are not explained by stimulus temporal structure}

To determine the extent to which the MEG signal could be explained by low-level responses to stimulus properties, we generated and correlated cochleograms pairwise from each stimulus condition. This analysis yielded an overall cochleogram-based dissimilarity curve (Fig. 6B) and, when performed on conditions pooled by source identity and space size, separate source and space dissimilarity curves (Fig. 6C). The cochleogram-based dissimilarity peaks (source, $495 \mathrm{~ms}$; space, $795 \mathrm{~ms}$ ) were significantly mismatched with the MEG decoding peaks $(p$ $<0.001$ for both source ${ }^{m}$ and space $^{n}$ via comparing to bootstrapped MEG peak latencies), a disparity that suggests the neural signal reflects processing other than the temporal structure of the stimulus.

\section{Reverberant spaces are encoded in a stepwise size pro-} gression

The MEG space decoding results shown in Figure $2 B$ could be suggestive of an encoded size scale (i.e., a small-to-large progression), or they could simply reflect a generic category difference between the three space conditions. To evaluate whether MEG responses were consistent with ordinal versus categorical spatial extent coding, we devised simple models of space representation in the form of RDMs that reflected the hypothesized space extent representations. That is, each pairwise representational distance in the model $9 \times 9$ condition matrix was either 0 or 1 , reflecting a within versus between separation (categorical space model), or 0,1 , or 2 reflecting a pure ordinal separation between space conditions respective of sound-source identity (ordinal space model). 
A Stimulus cochleograms

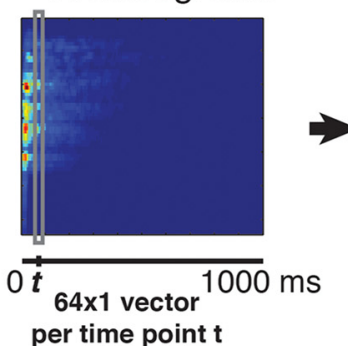

B

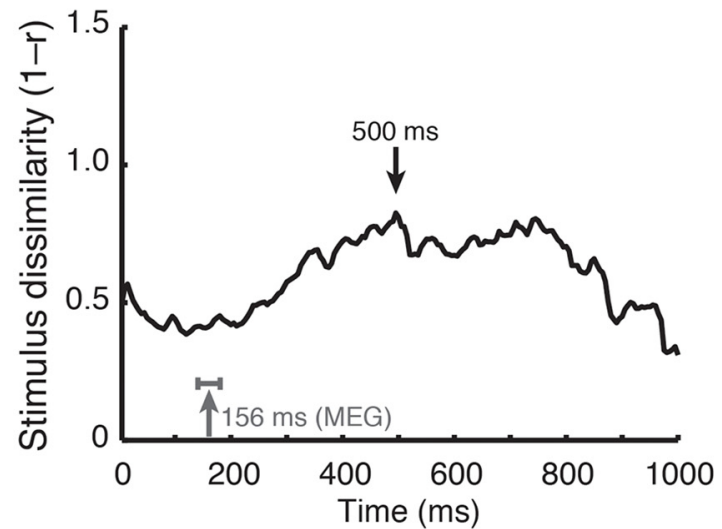

Cochleogram

dissimilarity matrix

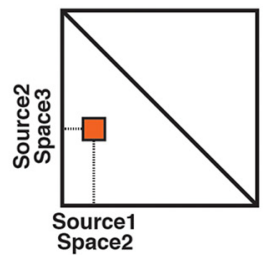

All pairwise comparisons
Dissimilarity

time course

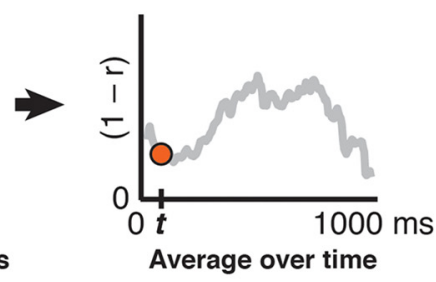

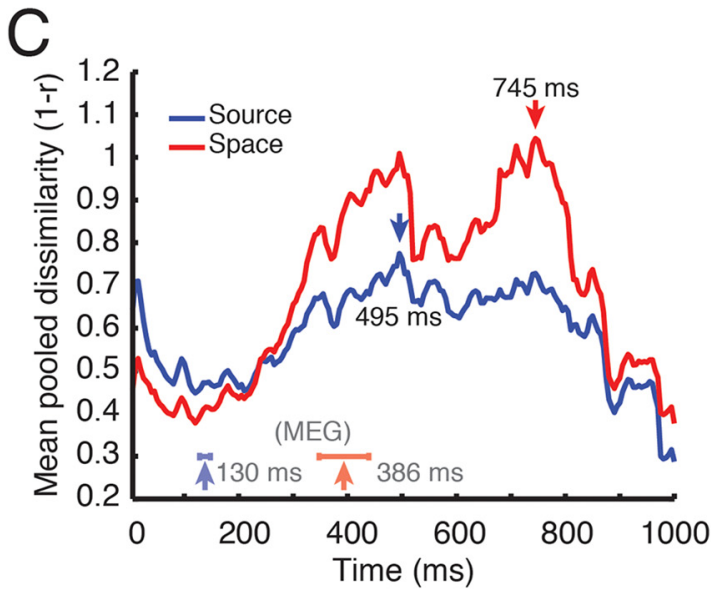

Figure 6. Stimulus dissimilarity analysis based on cochleogram data. $\boldsymbol{A}$, Cochleograms were generated for each stimulus, discretized into 2005 -ms bins and 64 frequency subbands. Each cochleogram thus comprised $20064 \times 1$ pattern vectors. For each pair of stimuli, pattern vectors across frequency subbands were correlated at corresponding time points and subtracted from 1. B, Overall cochleogram-based dissimilarity. The final dissimilarity value at time $t$ is an average of all pairwise correlations at that time point. Peak overall cochleogram dissimilarity occurred at $500 \mathrm{~ms}$; peak MEG dissimilarity (decoding accuracy) is shown for comparison. C, Pooled cochleogram-based dissimilarity across space size and source identity. Pairwise correlations were performed and averaged analogously to pooled decoding analysis. MEG pooled decoding peaks for source identity and space size are shown for reference; corresponding stimulus dissimilarity peaks were significantly offset $(p<0.05$ for both source identity and space).

We then correlated (using Spearman rank to capture ordinal relationships) the model RDMs with the brainresponse RDMs at every time point between the first and last time point of significant space decoding in the pooled analysis (138-801 ms poststimulus onset). Figure 4 shows that an ordinal space model correlates significantly more strongly with the neural data than a categorical model (663 time points, paired $t$ test, $p<0.00001)^{\circ}$, suggesting an ordinal representation of spatial size.

\section{Experiment 2}

Temporally extended stimuli elicit decoding dynamics similar to those of single-pulse stimuli

To examine whether peak decoding latencies reflect the timing of a neural operation or depend strictly on stimulus properties, we conducted a second MEG experiment examining the effect of longer (2000 ms) stimulus durations on decoding latencies. As shown in Figure $8 B$, soundsource decoding peaked at $167 \mathrm{~ms}(96-312 \mathrm{~ms})^{\mathrm{p}}$, while space decoding peaked at $237 \mathrm{~ms}(71-790 \mathrm{~ms})^{\text {q }}$. Responses remained significant throughout much of the stimulus duration but peaked early on, despite the amplitude envelope peaking in the middle of the stimulus, 1000 ms postonset. Thus, the neuromagnetic decoding signal reflects processing dissociable from the source and space information distributed throughout the longer stimulus.

\section{Discussion}

We investigated the neural representation of transient sound sources and their reverberant environments using multivariate pattern analysis (Carlson et al., 2013; Cichy et al., 2014) on MEG brain responses to spatialized sounds. Our results showed that overall individual sound conditions were decoded starting at $\sim 60 \mathrm{~ms}$ poststimulus onset, peaking at $156 \mathrm{~ms}$. Next, we characterized the separate neural time courses of source- and RIR-specific discrimination. These decoding profiles emerged with markedly different time courses, with the source discrimination time course exhibiting a rapid-onset transient response peaking at $130 \mathrm{~ms}$, and the space discrimination time course ramping up more gradually to peak at $386 \mathrm{~ms}$. Generalization of the responses across low-level variations was revealed by a cross-classification analysis in which training and testing trials contained different experimental factors. This suggests that these representations are tolerant to differences in amplitude envelope and spectral distributions, which accompany environmental 
A
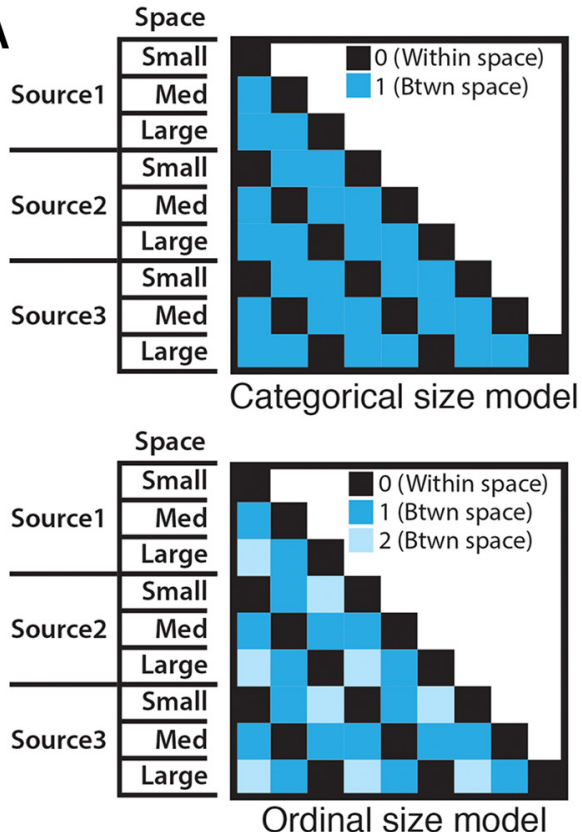

$\mathbf{B}$

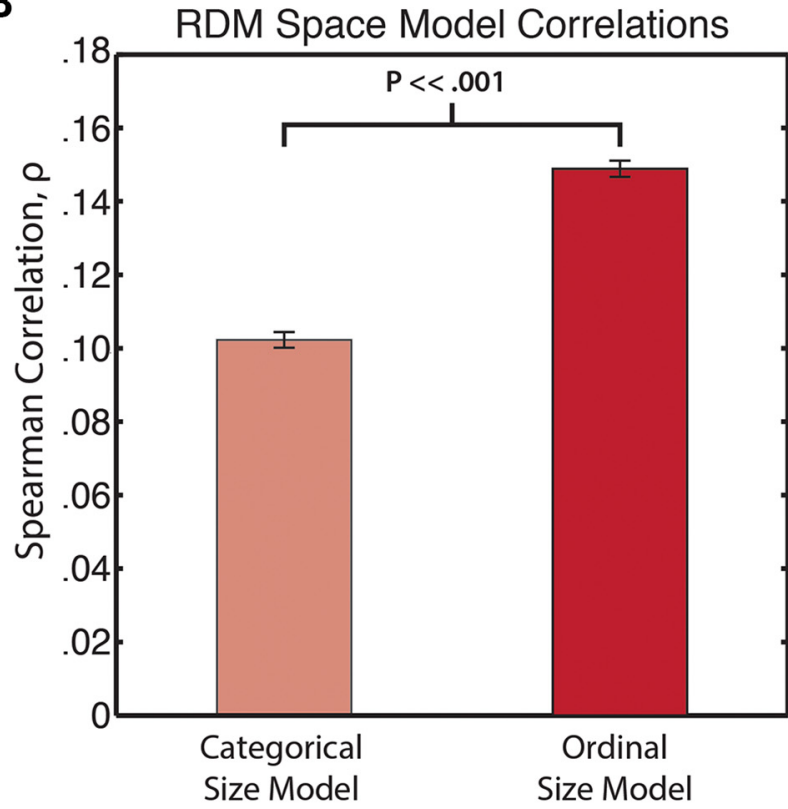

Figure 7. Comparison of MEG neural representations to a categorical versus an ordinal scene size model. Representational dissimilarity matrices (RDMs) of a categorical and an ordinal model $(\boldsymbol{A})$ were correlated with the MEG data from 138-801 ms (the temporal window of significant space size decoding) to assess the nature of MEG scene size representations. $\boldsymbol{B}$, Results indicate the MEG representations have significantly higher correlation with the ordinal than the categorical scene size model. Spearman correlation coefficients $\rho$ were averaged across time points in the temporal window. Error bars represent \pm SEM.

changes commonly encountered in real-world situations. A sensorwise decoding analysis showed that bilateral temporal cortical areas contributed most heavily to the decoding performance. MEG decoding peaks were significantly correlated with behavioral responses collected separately. Finally, the MEG decoding signal did not share the temporal profile of interstimulus correlations and peaked at similar times even when the stimulus was temporally extended, suggesting that it is separate from the stimulus temporal structure. Taken together, the results suggest that the MEG decoding time series capture the neural processes mediating extraction of sound source and reverberant information from naturalistic stimuli.
A

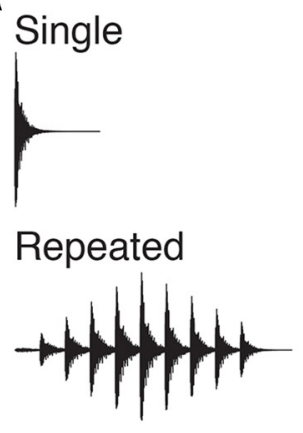

B

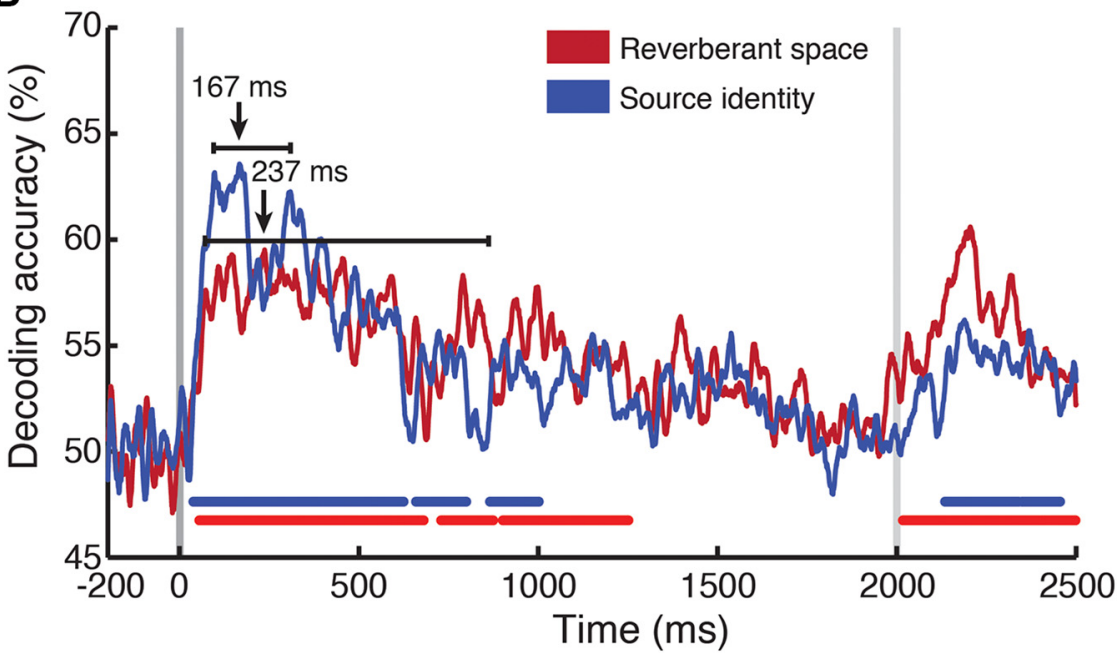

Figure 8. Space and sound source decoding with repetition-window stimuli. $\boldsymbol{A}$, Representative waveforms of single and repeated stimuli. Repeated stimuli were produced by concatenation of anechoic stimuli, followed by RIR convolution and linear amplitude ramping. B, Source (blue) and space (red) decoding. Sound-source classification peaked at 167 (96-312) ms, while space classification peaked at 237 (71-790) ms. Color-coded lines below time courses indicate significant time points, as in experiment 1; latency error bars indicate bootstrapped confidence intervals as in experiment 1. Gray vertical lines indicate stimulus onset and approximate offset. 


\section{Dissociable and independent decoding time courses}

The pooled and cross-classified source and space decoding time courses (Figs. 2, 3) indicate that reverberant space and sound-source representations are dissociable and independent in the brain. The early sound-source decoding peak suggests that source or object identity information is primarily carried by the direct sound and accompanying early reflections (the first, most nearby surface echoes; Hameed et al., 2004). By contrast, the later space decoding peak and concurrent falloff in source decoding suggest that reverberant decay primarily carries spatial extent information. This is broadly consistent with physical properties of large spaces, i.e., the longer propagation time for reflections to reach the listener, as well as longer $\mathrm{RT}_{60}$ reverberant decay times, in large spaces. However, even when source and space information was distributed throughout the stimuli equated for duration in experiment 2 , decoding peaks maintained a similar window of absolute and relative timing (Fig. 8), suggesting a consistent neural basis for the decoding time courses. However, testing other temporally extended nonimpulsive stimuli (e.g., speech, other environmental or synthetic sounds) constitutes a fruitful avenue for future work.

\section{Bilateral temporal decoding loci}

We observed a bilateral temporal decoding response to the stimulus conditions for space sizes as well as soundsource identities. While the spatial resolution of the sensorwise analysis cannot determine the exact loci of the signal sources, our results are consistent with a bilateral (Alain et al., 2001; Arnott et al., 2004) account of a spatial auditory processing stream, distinguishable from auditory object identification as early as nonprimary auditory cortical regions (Ahveninen et al., 2006, 2013). Room size judgments have been found to be correlated with soundsource distance judgments (Kolarik et al., 2013); while this may suggest a shared mechanism, it is unlikely to indicate distance as a direct proxy for size: the impulse responses in our stimuli kept sound-source distance constant, and the right-lateralized temporal processing of egocentric distance (Mathiak et al., 2003) is inconsistent with the bilateral decoding pattern in our results. Still, a source/ reverberant space separation operation by the auditory system would facilitate computing DRR for distance perception. Our data do not address this question directly but, along with Traer and McDermott (2016), suggest an intriguing counterpoint to interpretations that DRR computation is ill-posed and thus unlikely (Kopco and ShinnCunningham, 2011), or is bypassed via perception of other covarying cues (Larsen et al., 2008).

It has been speculated (Epstein, 2011) that spatial auditory scenes may be processed by the retrosplenial complex (RSC) and parahippocampal place area (PPA), occipital and ventral temporal brain regions known to be responsive to visually and haptically presented scene attributes (Wolbers et al., 2011; Park et al., 2015). The present sensorwise analysis most clearly implicates auditory-specific cortices in coding space size, although it does not preclude RSC and PPA. In addition to further examining this question, future work could compare the neural responses of blind and sighted listeners: people who are blind or visually impaired tend to weight echoes more heavily in perceiving their environments, whether passively listening (Després et al., 2005; Dufour et al., 2005) or actively echolocating (Teng et al., 2012; Kolarik et al., 2014; Thaler, 2015). Thus, given increased reliance on auditory information in blindness, frameworks of neuroplasticity espousing fundamental modality independence in neural function (Peelen and Kastner, 2009) suggest that RSC similarly represents auditory spatial scenes in blind persons.

\section{Dynamics in relation to previous electrophysiological work}

Most prior neuroimaging work on spatial auditory perception, including studies that used reverberant stimuli, measured brain responses to sound-source properties, rather than properties of the enclosing space. The spaceand source-decoding peak latencies found here are similar to that of numerous evoked neuromagnetic responses such as the mismatch negativity (Mathiak et al., 2003; King et al., 2014) and N1m response (Palomäki et al., 2005). This generally suggests that the neural activity underlying these evoked components may also be driving the source-specific MEG decoding performance, while low-level, preattentive-evoked responses with shorter latencies, such as the P50 (Mathiak et al., 2003), do not reflect neural activity that distinguishes among the spatial conditions, despite overlapping origins in bilateral temporal cortex (Liégeois-Chauvel et al., 1994). Later responses to naturalistic spatial stimuli include elevation-related processing starting at $200 \mathrm{~ms}$ (Fujiki et al., 2002) and a component indexing 3D "spatiality" of sound sources (Tiitinen et al., 2006), a reverberation-mediated parameter that may share similar underlying mechanisms with the space decoding signal in our results.

\section{The metrics of space representation}

While behavioral room size judgments have been previously shown to be driven by reverberant information, the precise relationship between volumetric spatial extent, reverberation time, and perceived room size is nonlinear and not fully understood (Mershon and King, 1975; Hameed et al., 2004; Kaplanis et al., 2014). We used a well-discriminable sequence of impulse responses from differently sized real-world spaces to establish an ordinal representation of spatial extent, but future work can more precisely characterize the metric by which the brain encodes auditory spatial extent and the interactions of monaural and binaural components of the signal. Future work may also more precisely map different acoustic components of the space signal to different neural correlates of perception, thus far explored almost exclusively in the behavioral realm (Kaplanis et al., 2014; but see Lawless and Vigeant, 2015). While spatial hearing is often considered inherently binaural, spatial properties carried by reverberation are not always so: diotic monaural stimuli have been used to judge spaces (Berkley and Allen, 1993; Traer and McDermott, 2016) and may in fact provide more salient spatial information than when listening binaurally (Shinn-Cunningham and Ram, 2003). Even when using 
binaural impulse responses, monaural cues in the sound are among the strongest predictors of perceived room size judgments (Pop and Cabrera, 2005; Zahorik, 2009). Still, binaural aspects of reverberation contain cues to spatially relevant percepts, such as spaciousness and apparent source width (Kaplanis et al., 2014), that our data do not address, and binaural listening may "squelch" or suppress perceived reverberation (Gelfand and Hochberg, 1976; but see Ellis et al., 2015). Finally, our RIRs were chosen from a set with $\mathrm{RT}_{60}$ averaging $<1 \mathrm{~s}$, and we did not explore effects of much longer times, which tend to correspond to larger spaces. Thus, our reverberant diotic stimuli likely reflect a subset of possible salient spatial extent cues that a real-world listener would encounter.

We note that space size as operationalized here is best defined for indoor environments; outdoor environments also have reverberant impulse responses, but their utility for judging spatial scale per se (vs sound-source distance; cf. Zahorik, 2002) is unclear. Although spatial layout can be recovered computationally from a few initial echoes (Dokmanic et al., 2013), human observers do not seem to have access to this information in real-world spaces (Calleri et al., 2016). Even in the visual scene and animal literatures, outdoor spaces have not been operationalized consistently (Kravitz et al., 2011; Park et al., 2011; Gevasagiv et al., 2015; Vanderelst et al., 2016), and thus the scene (and scene size) construct in general remains a topic for further research. However, recent work in scene processing has established that visual environments are represented along separable and complementary dimensions of spatial boundary and content (Kravitz et al., 2011; Park et al., 2011, 2015; Vaziri et al., 2014; Cichy et al., 2016). Thus, a visual scene may be characterized by, e.g., its encompassing shape and size, as well as by the number, type, and configuration of objects it contains (Oliva and Torralba, 2001; Oliva, 2013). Given that both visual and haptic scene exploration elicits responses in sceneselective brain regions (Wolbers et al., 2011), it is reasonable to surmise (cf. Epstein, 2011) some multimodality of scene representation. As a reliable index of perceived auditory scene extent (i.e., room size), natural reverberation could thus trigger scene-specific processing in a temporal regime that overlaps with those reported in recent M/EEG studies of visual scenes (Cichy et al., 2016; Harel et al., 2016).

In sum, the current study presents the first neuromagnetic evidence for the separation of auditory scenes into source and reverberant space representations in the brain. The neurodynamic profile of the processing stream is dissociable from that of sound sources in the scene, robust to variations in those sound sources, and predicts both timing and accuracy of corresponding behavioral judgments. Our results establish an auditory basis for neuroscientific investigations of scene processing, suggest the spatial importance of the reverberant decay in perceived scene properties, and lay the groundwork for future auditory and multisensory studies of perceptual and neural correlates of environmental geometry.

\section{References}

Ahveninen J, Huang S, Nummenmaa A, Belliveau JW, Hung A-Y, Jääskeläinen IP, Rauschecker JP, Rossi S, Tiitinen H, Raij T (2013) Evidence for distinct human auditory cortex regions for sound location versus identity processing. Nat Commun 4:2585. CrossRef Medline

Ahveninen J, Jääskeläinen IP, Raij T, Bonmassar G, Devore S, Hämäläinen $M$, Levänen S, Lin F-H, Sams M, Shinn-Cunningham BG, Witzel T, Belliveau JW (2006) Task-modulated "what" and "where" pathways in human auditory cortex. Proc Natl Acad Sci USA 103:14608-14613. CrossRef Medline

Alain C, Arnott SR, Hevenor S, Graham S, Grady CL (2001) "What" and "where" in the human auditory system. Proc Natl Acad Sci USA 98:12301-12306. CrossRef Medline

Arnott SR, Binns MA, Grady CL, Alain C (2004) Assessing the auditory dual-pathway model in humans. Neuroimage 22:401-408. CrossRef Medline

Berkley DA, Allen JB (1993) Normal listening in typical rooms: the physical and psychophysical correlates of reverberation. In: Acoustical factors affecting hearing aid performance (Studebaker GA, Hockberg I, eds), pp 3-14. Boston: Allyn and Bacon.

Bizley JK, Cohen YE (2013) The what, where and how of auditoryobject perception. Nat Rev Neurosci 14:693-707. CrossRef Medline

Blauert J (1997) Spatial hearing. Cambridge, MA: MIT Press.

Brandewie E, Zahorik P (2010) Prior listening in rooms improves speech intelligibility. J Acoust Soc Am 128:291-299. CrossRef Medline

Bregman AS (1994) Auditory scene analysis: the perceptual organization of sound. Cambridge: MIT Press.

Bronkhorst AW, Houtgast T (1999) Auditory distance perception in rooms. Nature 397:517-520. CrossRef Medline

Brown AD, Jones HG, Kan AH, Thakkar T, Stecker GC, Goupell MJ, Litovsky RY (2015) Evidence for a neural source of the precedence effect in sound localization. J Neurophysiol 11:2291-3001.

Cabrera D, Pop C (2006) Auditory room size perception: a comparison of real versus binaural sound-fields. Proceedings of the 1st Australasian Acoustic Societies' Conference, pp 417-422. Christchurch, New Zealand.

Calleri C, Astolfi A, Armando A, Shtrepi L (2016) On the ability to correlate perceived sound to urban space geometries. Sustain Cities Soc 27:346-355.

Carlson T, Tovar D, Alink A, Kriegeskorte N (2013) Representational dynamics of object vision: the first $1000 \mathrm{~ms}$. J Vis 13:1-19. CrossRef

Chang C-C, Lin C-J (2011) LIBSVM. A library for support vector machines. ACM Trans Intell Syst Technol 2:1-27. CrossRef

Cichy RM, Khosla A, Pantazis D, Oliva A (2016) Dynamics of scene representations in the human brain revealed by magnetoencephalography and deep neural networks. Neuroimage pii:S10538119(16)30007-6.

Cichy RM, Pantazis D, Oliva A (2014) Resolving human object recognition in space and time. Nat Neurosci 17:455-462. CrossRef Medline

Cichy RM, Ramirez FM, Pantazis D (2015) Can visual information encoded in cortical columns be decoded from magnetoencephalography data in humans? Neuroimage 121:193-204. CrossRef Medline

Després O, Candas V, Dufour A (2005) The extent of visual deficit and auditory spatial compensation: evidence from self-positioning from auditory cues. Cogn Brain Res 23:444-447. CrossRef Medline

Devore S, Ihlefeld A, Hancock K, Shinn-Cunningham B, Delgutte B (2009) Accurate sound localization in reverberant environments is mediated by robust encoding of spatial cues in the auditory midbrain. Neuron 62:123-134. CrossRef Medline

Dokmanic I, Parhizkar R, Walther A, Lu YM, Vetterli M (2013) Acoustic echoes reveal room shape. Proc Natl Acad Sci USA 110: 12186-12191. CrossRef Medline 
Dufour A, Després O, Candas V (2005) Enhanced sensitivity to echo cues in blind subjects. Exp Brain Res 165:515-519. CrossRef Medline

Ellis DPW (2009) Gammatone-like spectrograms. Available at http:// www.ee.columbia.edu/ dpwe/resources/matlab/gammatonegram/ [Accessed June 5, 2016].

Ellis GM, Zahorik P, Hartmann WM (2015) Using multidimensional scaling techniques to quantify binaural squelch. J Acoust Soc Am 137:2231-2231.

Epstein RA (2011) Cognitive neuroscience: scene layout from vision and touch. Curr Biol 21:R437-R438. CrossRef Medline

Fujiki N, Riederer KAJ, Jousmäki V, Mäkelä JP, Hari R (2002) Human cortical representation of virtual auditory space: differences between sound azimuth and elevation. Eur J Neurosci 16:2207-2213. Medline

Gelfand SA, Hochberg I (1976) Binaural and monaural speech discrimination under reverberation. Audiology 15:72-84. Medline

Geva-sagiv M, Las L, Yovel Y, Ulanovsky N (2015) Spatial cognition in bats and rats: from sensory acquisition to multiscale maps and navigation. Nat Rev Neurosci 16:94-108. CrossRef Medline

Hameed S, Pakarinen J, Valde K, Pulkki V (2004) Psychoacoustic cues in room size perception. 116th Convention of the Audio Engineering Society, pp 1-7. Berlin, Germany.

Harel A, Groen IIA, Kravitz DJ, Deouell LY, Baker CI (2016) The temporal dynamics of scene processing: a multi-faceted EEG investigation. eNeuro 3.

Kaplanis N, Bech S, Jensen SH, Van Waterschoot T (2014) Perception of reverberation in small rooms: a literature study. Presented at the 55th International Conference: Spatial Audio, Audio Engineering Society, pp 0-14. Helsinki, Finland.

King JR, Dehaene S (2014) Characterizing the dynamics of mental representations: the temporal generalization method. Trends Cogn Sci 18:203-210. CrossRef Medline

King J-R, Gramfort A, Schurger A, Naccache L, Dehaene S (2014) Two distinct dynamic modes subtend the detection of unexpected sounds. PLoS One 9:e85791. CrossRef Medline

Kolarik AJ, Cirstea S, Pardhan S, Moore BCJ (2014) A summary of research investigating echolocation abilities of blind and sighted humans. Hear Res 310:60-68. CrossRef Medline

Kolarik AJ, Moore BCJ, Zahorik P, Cirstea S (2016) Auditory distance perception in humans: a review of cues, development, neuronal bases, and effects of sensory loss. Atten Percept Psychophys 78:373-395. CrossRef

Kolarik AJ, Pardhan S, Cirstea S, Moore BCJ (2013) Using acoustic information to perceive room size: effects of blindness, room reverberation time, and stimulus. Perception 42:985-990. Medline

Kopco N, Shinn-Cunningham BG (2011) Effect of stimulus spectrum on distance perception for nearby sources. J Acoust Soc Am 130:1530-1541.

Kravitz DJ, Peng CS, Baker Cl (2011) Real-world scene representations in high-level visual cortex: it's the spaces more than the places. J Neurosci 31:7322-7333. CrossRef Medline

Kriegeskorte N, Mur M, Bandettini P (2008) Representational similarity analysis - connecting the branches of systems neuroscience. Front Syst Neurosci 2:4. CrossRef Medline

Larsen E, Lansing C, Feng AS (2008) On the minimum audible difference in direct-to- reverberant energy ratio. J Acoust Soc Am 124:450-461. CrossRef Medline

Lawless MS, Vigeant MC (2015) Investigating the emotional response to room acoustics: a functional magnetic resonance imaging study. J Acoust Soc Am 138:417-423. CrossRef

Liégeois-Chauvel C, Musolino A, Badier JM, Marquis P, Chauvel P (1994) Evoked potentials recorded from the auditory cortex in man: evaluation and topography of the middle latency components. Electroencephalogr Clin Neurophysiol 92:204-214. CrossRef

Litovsky RY, Colburn HS, Yost WA, Guzman SJ (1999) The precedence effect. J Acoust Soc Am 106:1633-1654. Medline

Lokki T, Patynen J, Kuusinen A, Vertanen H, Tervo S (2011) Concert hall acoustics assessment with individually elicited attributes. $\mathrm{J}$ Acoust Soc Am 130:835. CrossRef Medline
Maris E, Oostenveld R (2007) Nonparametric statistical testing of EEG- and MEG-data. J Neurosci Methods 164:177-190. CrossRef Medline

Mathiak K, Hertrich I, Kincses WE, Riecker A, Lutzenberger W, Ackermann H (2003) The right supratemporal plane hears the distance of objects: neuromagnetic correlates of virtual reality. Neuroreport 14:307-311. CrossRef Medline

McGrath R, Waldmann T, Fernström M (1999) Listening to rooms and objects. 16th Audio Engineering Society International Conference on Spatial Sound Reproduction. Rovaniemi, Finland.

Mershon DH, King LE (1975) Intensity and reverberation as factors in the auditory perception of egocentric distance. Percept Psychophys 18:409-415. CrossRef

Mesgarani N, David SV, Fritz JB, Shamma SA (2014) Mechanisms of noise robust representation of speech in primary auditory cortex. Proc Natl Acad Sci USA 111:6792-6797. CrossRef Medline

Middlebrooks JC, Green DM (1991) Sound localization by human listeners. Annu Rev Psychol 42:135-159. CrossRef Medline

Oliva A (2013) Scene perception. In: The new visual neurosciences. (Werner JS, Chalupa LM, eds), pp 725-732. Cambridge: MIT Press.

Oliva A, Torralba A (2001) Modeling the shape of the scene: a holistic representation of the spatial envelope. Int J Comput Vis 42:145175. CrossRef

Palmer EM, Horowitz TS, Torralba A, Wolfe JM (2011) What are the shapes of response time distributions in visual search? J Exp Psychol Hum Percept Perform 37:58-71. CrossRef Medline

Palomäki KJ, Tiitinen H, Mäkinen V, May PJC, Alku P (2005) Spatial processing in human auditory cortex: the effects of 3D, ITD, and ILD stimulation techniques. Cogn Brain Res 24:364-379. CrossRef Medline

Park S, Brady TF, Greene MR, Oliva A (2011) Disentangling scene content from spatial boundary: complementary roles for the parahippocampal place area and lateral occipital complex in representing real-world scenes. J Neurosci 31:1333-1340. CrossRef Medline

Park S, Konkle T, Oliva A (2015) Parametric coding of the size and clutter of natural scenes in the human brain. Cereb Cortex 25: 1792-1805. CrossRef Medline

Peelen MV, Kastner S (2009) A nonvisual look at the functional organization of visual cortex. Neuron 63:284-286. CrossRef Medline

Pop CB, Cabrera D (2005) Auditory room size perception for real rooms. Proc Acoust 2005:115-121.

Shinn-Cunningham B (2003) Acoustics and perception of sound in everyday environments. In: Proc of 3rd International Workshop on Spatial Media, Aizu-Wakamatsu, Japan.

Shinn-Cunningham BG, Ram S (2003) Identifying where you are in a room: sensitivity to room acoustics. Proceedings of the 2003 International Conference on Auditory Display, pp 21-24. Boston, MA.

Slaney M (1998) Auditory toolbox version 2. Technical report \#1998010.

Stecker GC, Hafter ER (2000) An effect of temporal asymmetry on loudness. J Acoust Soc Am 107:3358-3368. Medline

Tadel F, Baillet S, Mosher JC, Pantazis D, Leahy RM (2011) Brainstorm: a user-friendly application for MEG/EEG analysis. Comput Intell Neurosci 2011:879716. CrossRef

Taulu S, Kajola M, Simola J (2004) Suppression of interference and artifacts by the signal space separation method. Brain Topogr 16:269-275. Medline

Taulu S, Simola J (2006) Spatiotemporal signal space separation method for rejecting nearby interference in MEG measurements. Phys Med Biol 51:1759-1768. CrossRef Medline

Teng S, Puri A, Whitney D (2012) Ultrafine spatial acuity of blind expert human echolocators. Exp Brain Res 216:483-488. CrossRef Medline

Thaler L (2015) Using sound to get around. Observer .

Tiitinen H, Salminen NH, Palomäki KJ, Mäkinen VT, Alku P, May PJC (2006) Neuromagnetic recordings reveal the temporal dynamics of auditory spatial processing in the human cortex. Neurosci Lett 396:17-22. CrossRef 
Traer J, McDermott JH (2016) Statistics of natural reverberation enable perceptual separation of sound and space. Proc Natl Acad Sci USA 113:E7856-E7865. CrossRef Medline

Vanderelst D, Steckel J, Boen A, Peremans H, Holderied MW (2016) Place recognition using batlike sonar. Elife 5.pii:e14188.

Vaziri S, Carlson ET, Wang Z, Connor CE (2014) A channel for 3D environmental shape in anterior inferotemporal cortex. Neuron 84:55-62. CrossRef Medline

Watkins AJ, Raimond AP (2013) Perceptual compensation when isolated test words are heard in room reverberation. In: Basic aspects of hearing: physiology and perception (Moore BCJ, Patterson RD, Winter IM, Carlyon RP, Gockel HE, eds), pp 193-202. San Diego: Springer.

Wolbers T, Klatzky RL, Loomis JM, Wutte MG, Giudice NA (2011) Modality-independent coding of spatial layout in the human brain. Curr Biol 21:984-989. CrossRef Medline
Zahorik P (2001) Estimating sound source distance with and without vision. Optom Vis Sci 78:270-275. Medline

Zahorik P (2002) Assessing auditory distance perception using virtual acoustics. J Acoust Soc Am 111:1832-1846. Medline

Zahorik P (2009) Perceptually relevant parameters for virtual listening simulation of small room acoustics. J Acoust Soc Am 126:776791. CrossRef Medline

Zahorik P, Brungart DS, Bronkhorst AW (2005) Auditory distance perception in humans: a summary of past and present research. Acta Acust United Acust 91:409-420.

Zahorik P, Wightman FL (2001) Loudness constancy with varying sound source distance. Nat Neurosci 4:78-83. CrossRef Medline

Zhou B, Green DM, Middlebrooks JC (1992) Characterization of external ear impulse responses using Golay codes. J Acoust Soc Am 92:1169-1171. Medline 